\section{Sub-bandage dynamics: stiffness unravelled}

\author{
Jan Schuren, ${ }^{1}$ Jens Bichel ${ }^{2}$ \\ ${ }^{1}$ Retired employee of; ${ }^{23}$ Meutschland \\ GmbH, Neuss, Germany
}

\section{Abstract}

The static stiffness index (SSI) is mathematical equation that results in a simple number when the sub-bandage pressure in the supine position is subtracted from the subbandage pressure in the standing weight-bearing position. When SSI data are reported, often a wide range of values is observed for similar materials. The aim of this study was to explore the strength and weakness of the SSI and its measurement. Pressure was recorded with bandaging materials with different resting pressures and properties. Measurements in the upright position were performed under weight and non-weight bearing conditions for up to 12 min of motionless stance. The measurements reveal that the SSI reveals more about the muscle forces of the person included in the system, rather than providing accurate information on the applied system or how well this system is applied. In addition, venous filling has a major effect on the final SSI. When performed under similar conditions, the SSI is able to differentiate between elastic and inelastic materials. The SSI gives us a rough estimate of the effectiveness of an applied system but interpretation is influenced by the muscle forces of the person being bandaged as well as the measured effects of venous filling and, because of that, the timing of the measurements. Future guidelines on measuring the SSI should include that the final standing pressure value should be taken when a stable recording over a certain period is observed.

\section{Introduction}

There is a variety of methods to describe the properties of bandaging materials. Recently a consensus document was published, in which was stated that sub-bandage pressures and material stiffness characterize the elastic properties of the used materials and are the deciding parameters determining the dosage of compression treatment. ${ }^{1}$ Therefore, it was recommended to measure and report these characteristics in future clinical trials. Proposals were made concerning methods for measuring the interface pressure and for assessing the stiffness of a compression device in an individual patient. However, stiff- ness is more than just a mathematical equation that results in a simple number. This article explores the strength and weakness of the static stiffness index (SSI).

\section{The B1-position}

In the European Committee for Standardization (CEN) Prestandard document, ${ }^{2}$ an overview is provided on the anatomical locations to position pressure sensors on a leg. One of these locations is called $\mathrm{cB} 1$, the area at which the Achilles tendon changes into the calf muscles, approximately $10-15 \mathrm{~cm}$ proximal to the medial malleolus. Stolk et al. ${ }^{3}$ performed static measurements and showed that the largest differences in the circumference between the maximal dorsiflexion and maximal plantar flexion positions of the foot occur at the level of the transition from the gastrocnemius muscle into its aponeurosis (the $\mathrm{cB1}$ level or simplified: B1; Figure 1). The International Compression Club (ICC) consensus document proposes that location B1 should always be included in future pressure measurements, with the exact location of the sensor situated at the segment that shows the most extensive enlargement of the leg circumference during dorsiflexion or by standing up from the supine position. ${ }^{1}$ Although B1 should always be included as a measurement location, other sites could be included in any measurement of pressures. ${ }^{1}$ Figure 1 shows a screenshot of measurements with the PicoPress device (Microlab Elettronica SAS, Ponte S. Nicolò, Italy) and the sensor positioned at the $\mathrm{B} 1$ position. The measured pressure values are marked A, B, C.

\section{Resting pressure, standing pres- sure, amplitudes}

The resting pressure gives an indication of how much pressure is provided by a compression system when the subject is in a relaxed supine position with a slightly flexed knee and the foot resting on a flat surface. It is important that the calf muscles are not resting on a surface, as the result may be a too high resting pressure. ${ }^{4}$ In Figure 1, the resting pressure (A) is around $40 \mathrm{mmHg}$.

The standing pressure gives an indication of the pressure when the subject is asked to stand up and put weight on the compressed leg. ${ }^{5,6}$ In Figure 1, the standing pressure (B) is around $70 \mathrm{mmHg}$.

Resting and standing pressure are both values recorded in static situations. If a measuring device (like e.g. PicoPress) allows dynamic recording, it is advisable to measure also the amplitudes of a specified movement.

Possible movements include the following: ${ }^{1}$ i) dorsal and plantar flexion of the ankle joint; ii) walking, for example on a treadmill; iii) adopting a tip-toe stance, or flexing of the
Correspondence: Jan Schuren, Grotestraat 34 6067 BR Linne, The Netherlands.

E-mail: jan.schuren@gmail.com

Key words: compression therapy, static stiffness index, working amplitudes, venous filling.

Conference presentation: part of this paper was presented at the International Compression Club (ICC) Meeting on Stiffness of Compression Devices, 2012 May 25, Vienna, Austria (http://www.icc-compressionclub.com/).

Contributions: JS, manuscript writing; the authors contributed equally to: conception and design; data acquisition, analysis and interpretation; final approval of the version to be published.

Conflict of interests: JS is a retired 3M employee and invented and co-developed the 3M Coban 2 Layer compression systems; JB is employed by $3 \mathrm{M}$.

Received for publication: 20 October 2012. Revision received: 22 November 2012. Accepted for publication: 29 November 2012.

This work is licensed under a Creative Commons Attribution 3.0 License (by-nc 3.0).

(C) Copyright J. Schuren and J. Bichel, 2013 Licensee PAGEPress, Italy

Veins and Lymphatics 2013; 2:e2 doi:10.4081/vl.2013.e2

knees; iv) passive ankle movement.

In Figure 1, the amplitudes are presented in the column exercise. The range of pressure values (C) is between 45 and $90 \mathrm{mmHg}$. The difference between these two pressure values results in a working pressure amplitude (WPA) The recording during the exercise in Figure 1 gives a WPA of 45 .

\section{The static stiffness index}

The CEN European Prestandard document for medical compression hosiery defines stiffness as the increase in pressure per $1 \mathrm{~cm}$ increase of leg circumference. ${ }^{2}$ For compression bandages, the extensibility of materials is often used to determine their characteristics. Partsch ${ }^{5}$ identified the need for a simple tool to assess both pressure and stiffness on the individual leg. He describes the method to measure the pressure at a defined position of the lower leg at rest (B1), when its circumference is minimal, and to repeat the measurement on the same spot, when the circumference has maximally increased by the muscles actively engaged to stand in the upright position. For measuring stiffness, the pressure in the supine position is subtracted from the pressure in stance. The resulting index indicates the effectiveness of the applied system. ${ }^{1}$ This index is referred to as SSI and, although it 
might be influenced by many variables, provides an indication of how well an applied compression system manages to keep forces produced by the muscle activity to stay in the upright position, inside the compressed area. In the measurement presented in Figure 1, a typical PicoPress recording is presented of the pressure under a $3 \mathrm{M}^{\mathrm{TM}}$ Coban ${ }^{\mathrm{TM}} 2$ Layer application (3M ${ }^{\mathrm{TM}}$ HealthCare, St. Paul, MN, USA), with the sensor positioned at the B1 location. The resting pressure is presented in the column supine and is around $40 \mathrm{mmHg}$ (A). The standing pressure can be taken from the column stance and is around $70 \mathrm{mmHg}$ (B). This means that the SSI in this measurement is 30 (70-40).

\section{Results and Discussion}

\section{Muscle forces}

It is easy to imagine that both SSI and WPA are not only determined by the stiffness of the applied compression system but more by the muscle forces that are produced inside the bandaged area. Provided that the measurements are not performed on a leg with major disfigurations due to severe obesity or lymphoedema, the subject inside the system heavily confounds each measurement. As a consequence of measuring the muscle forces inside the compression system, both SSI and WPA tell more about the muscle forces of the person included in the system, rather than providing accurate information on the applied system or how well this system is applied. This can be easily demonstrated with the measurements presented in Figure 2. With the same system applied in the same way by the same experienced bandager on different subjects, the amplitudes are 23 on the left (C: $55-32$ ) and 64 on the right pressure profile (C: 102-38).

These measurements are from a study on healthy volunteers, recorded with a Gaeltec strain gauge temperature-compensated (15$40^{\circ} \mathrm{C}$ ) force transducer (Gaeltec Devices Ltd, Dunvegan, Isle of Skye, UK). The transducer was positioned at the B1 position and connected to a computer from which the data was recorded. The only difference in the two recordings is the volunteer. In both readings, a similar resting pressure was achieved. The SSI's (14 versus 46 ) as well as the WPA's during walking on a treadmill (23 versus 61 ) of the used system show big differences. This phenomenon can also be observed in studies in which actual SSI measurements are presented. A few studies present data on measurements on short-stretch bandages. Partsch (Derm Surg 2005) presents data of measurements on 12 volunteers. The reported SSI values vary between 10 and >40 for both Unna's boot and multilayer short-stretch bandages. Similar differences in reported SSI's are observed in publications by Mosti et al. ${ }^{7,8}$ and Partsch et al. ${ }^{9}$ In some of these measurements, there is even an overlap of individual values from the systems with the highest and lowest mean stiffness (e.g. 7).

\section{The static stiffness index and venous filling}

Another factor that might influence the accuracy of the SSI is the timing of the measurements. There are no clear guidelines on when recording of the standing pressure

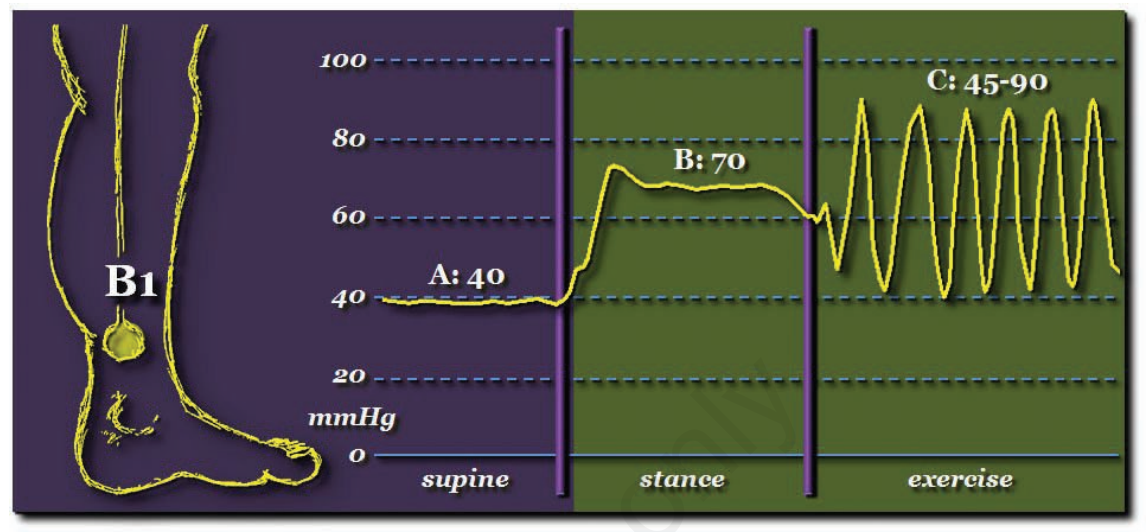

Figure 1. A typical PicoPress recording of a bandaged leg with the sensor positioned at the $B 1$ location shown on the left. The measurements show the pressure in the supine position (A), in the standing position (B) and during functional activities (C).

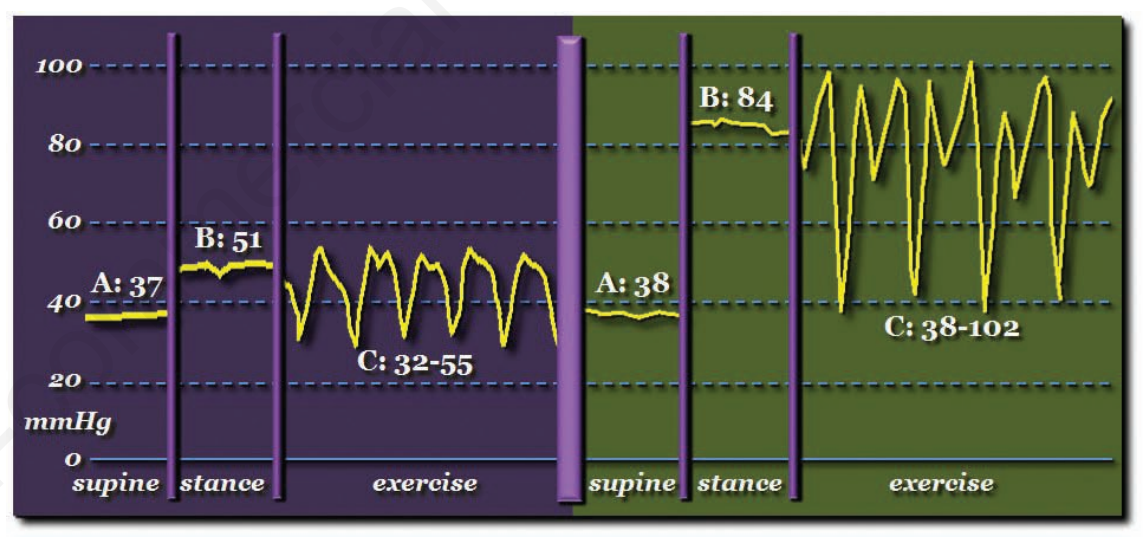

Figure 2. Sub-bandage pressure recordings from two different volunteers with the same compression system applied by the same experienced bandager.

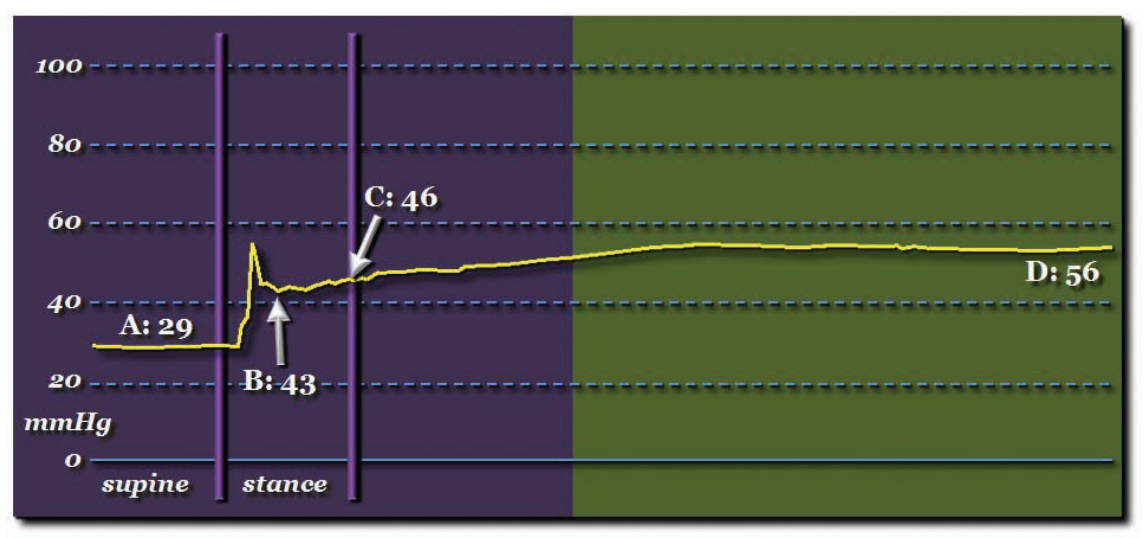

Figure 3. Recording of sub-bandage pressure of a normal limb in a Coban 2 Lite compression system, including the change from the supine to a weight bearing standing position. 
should be performed. Similar to a normal unwrapped leg, the venous filling of a bandaged leg takes a certain period. Nicolaides $e t$ al. ${ }^{10}$ recorded intravenous pressure of a normal limb in a vein on the dorsum of the foot. After ten tip-toe movements, it takes more than $30 \mathrm{~s}$ before the venous pressure returns to the pressure before the exercise. A similar refilling time can be observed after the application of a compression system, when the subject changes from a supine to an upright posture. Figure 3 provides an example of a healthy subject, compressed with Coban 2 Lite $\left(3 \mathrm{M}^{\mathrm{TM}}\right.$ HealthCare). Recording was performed immediately after the application. During the measurements in the upright position, the volunteer holds on to a frame to avoid balancing muscle activities in the leg. If the instructions of the used device (PicoPress) are followed, the pressure is taken from some of the values in the period located between the first two pink vertical lines. At the second line, the device gives a signal that the standing period is completed. Immediately after the position change, the standing pressure is $43 \mathrm{mmHg}$ (B); the pressure at the end of this period is $46 \mathrm{mmHg}$ (C). Looking at the resting pressure of 29 , a reported SSI could be between 14 and 17 . Venous filling of the lower limb however, takes much longer than the advised period. After the position change, it takes almost a minute before a stable pressure level of $56 \mathrm{mmHg}$ (D) can be observed. If that recording would be used for the calculation, the SSI would be 27 . The consequence of the above observations is that, depending on the time of measurement; the SSI can vary between 14 and 27 .

Figure 4 shows another recording of the same leg in the same bandage. The resting pressure is $30 \mathrm{mmHg}(\mathrm{A})$. Now the position change takes place without weight bearing. The volunteer steps on an elevation, bearing full weight on the contralateral leg. The bandaged leg is hanging free with a relaxed Achilles tendon. The initial pressure after this position change is $23 \mathrm{mmHg}$ (B) and $28 \mathrm{mmHg}$ after the signal $(C)$ of the device. As in the previous recording, it takes a minute before a final stable pressure is established. This final pressure is $48 \mathrm{mmHg}$ (D). During the change from the supine to the standing position, venous filling in isolation creates a pressure increase of 18 mmHg. In patients with chronic venous insufficiency, veins refill quickly and a stable recording can be observed much faster than in the provided example with a healthy volunteer. ${ }^{11}$ In addition, it might be assumed, that in patients with significant venous dilatation, pressure increase due to venous refilling is more pronounced than in healthy volunteers. This could be explained by higher volume increase of dilated veins in the upright position, until an increasing venous wall tension prevents further venous filling. This means that in patients with chronic venous insufficiency the right time of standing pressure measurement is even more important.

Pannier et al. ${ }^{12}$ measured the increase in leg volume increase after changing from a lying to a standing position and demonstrated that the position change initially leads to a rapid increase in volume. The main change is observed in the $1^{\text {st }} \mathrm{min}$, followed by a further slower increase in the next $9 \mathrm{~min}$. The authors state that the volume increase follows a biexponential function fitting to a rapid filling compartment (venous pooling) and a slow filling compartment-reflecting extravasation.

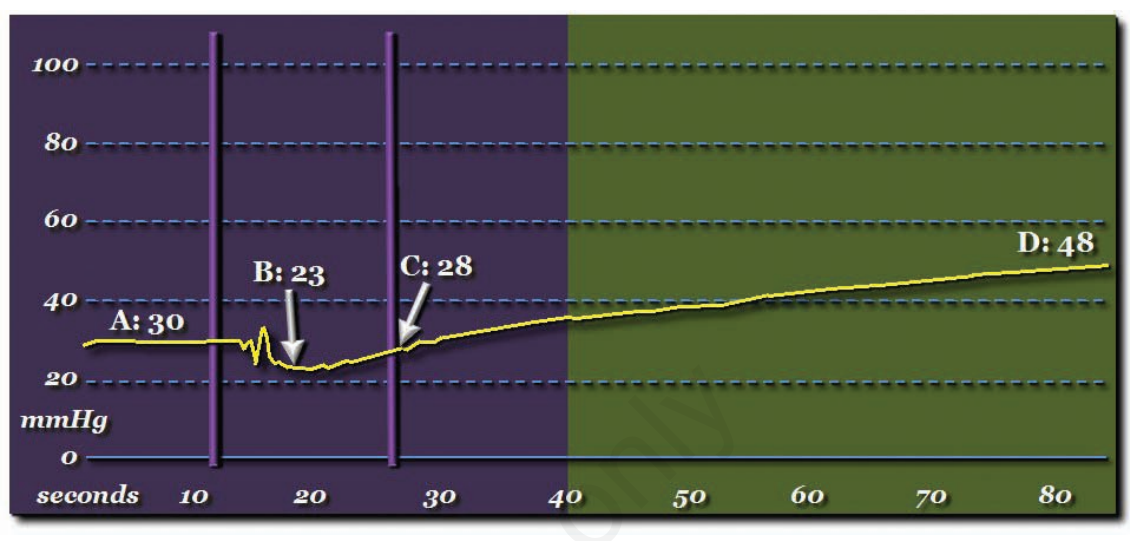

Figure 4. Recording of sub-bandage pressure of a normal limb under a Coban 2 Lite application including the change from a supine to a non-weight bearing standing position.

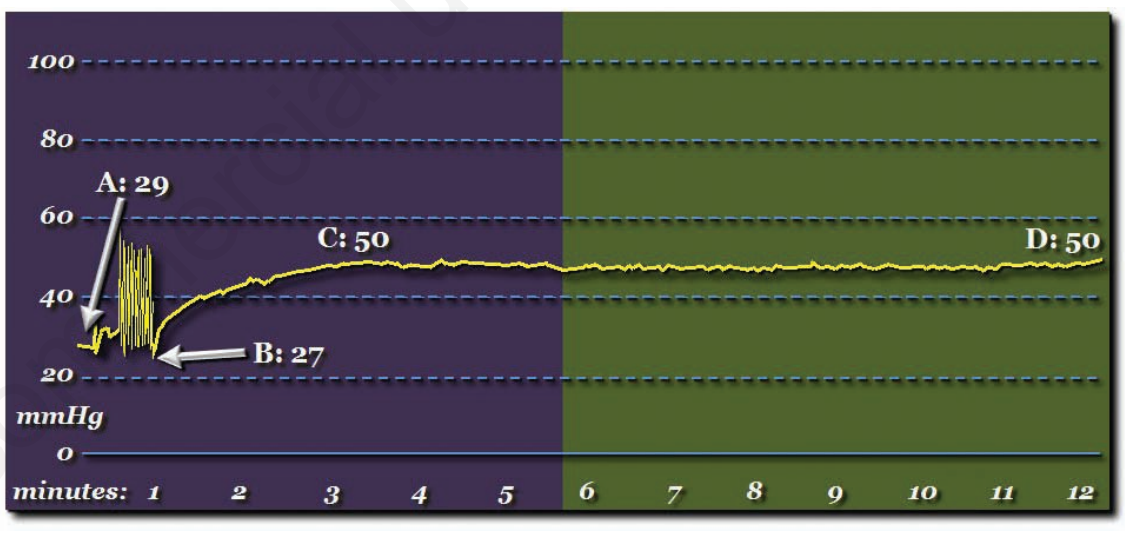

Figure 5. Recording of sub-bandage pressure of a normal limb compressed with Coban 2 Lite, including the change from the supine to non weight bearing standing position, which was maintained motionless during the entire recording time.

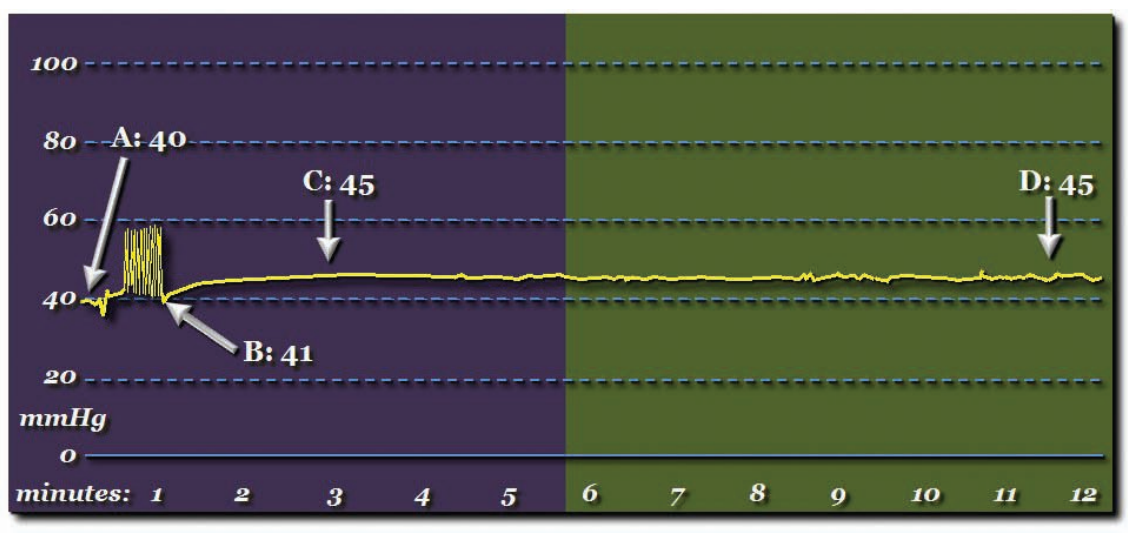

Figure 6. Recording of sub-bandage pressure of a normal limb compressed with a longstretch bandage, including the change from the supine to non weight bearing standing position, which was maintained motionless during the entire recording time.

[Veins and Lymphatics 2013; 2:e2]

[page 5] 
Stick et al. ${ }^{13}$ used strain gauge plethysmography at calf and ankle level to document the volume changes, which occurred when a subject was tilted from the supine to the upright position. In both ankle and calf, the highest volume increase was observed in the first $2 \mathrm{~min}$, after which the volume further increased at a less steep slope. The authors state that after the subject has been brought into the upright posture, an increased hydrostatic pressure in the arteries makes the blood flow via the arteriolar resistance vessels and via the capillaries into the venous capacitance vessels. Next, a further volume increase is observed in the following $10 \mathrm{~min}$, which is due to an increased transcapillary filtration of fluid into the interstitial space. Mosti et al. ${ }^{14}$ demonstrated that there is a significant correlation between the degree of improvement in venous hemodynamics of the ejection fraction (EF) examined by strain gauge plethysmography and both the SSI and the amplitudes of sub-bandage pressure during walking. The authors report that when elastic bandages are applied at high pressure and high stretch, only small pressure differences (SSI and WPA) occur by standing and walking resulting in low EF values. To evaluate the fluid shift into the interstitial space, we measured the effects of the position change on sub-bandage pressures during 12 min of standing with the leg under investigation in the non-weight bearing position. The subject is wearing the inelastic Coban 2 Lite compression system. As can be seen in Figure 5 , the initial resting pressure is $29 \mathrm{mmHg}$ (A). Next, the volunteer performed ten active maximal dorsal and plantar flexions. After the exercises, the pressure returned to $27 \mathrm{mmHg}$ (B), a little lower that the initial resting pressure. Similar to what was observed in Figure 4; venous filling brings the pressure to $50 \mathrm{mmHg}$ after 2.5 min (C). During the next 10 min of motionless stance, no change in pressure is observed (D: 50). This means that the bandage, which was applied at full stretch, manages to keep the forces that are generated by the dorsal and plantar flexions, inside the system, as well as the forces generated by the venous refilling. However, because the forces needed for the interstitial fluid shift into the lower leg (edema) are much lower than the gravitational forces responsible for venous refilling, it can be hypothesized that compression applied at full stretch also provides a sufficient counterforce for the forces responsible for the interstitial fluid shift, as they are not high enough to generate an additional increase of sub-bandage pressure $(\mathrm{C}=\mathrm{D})$.

This procedure was repeated after the application of the long-stretch compression bandage Biflex 16+ (Thuasne SA, Levallois Perret, France) with tension indicators for accuracy of application; the tension is correct when the printed markers are square-shaped. The bandage was applied in a spica manner according the included manufacturers instructions for use. The recording of this application is presented in Figure 6. The application provides a resting pressure of around $40 \mathrm{mmHg}$ (A). After the exercises, the pressure is $41 \mathrm{mmHg}$ (B). Venous filling brings the pressure to 45 $\mathrm{mmHg}$ after $2 \mathrm{~min}(\mathrm{C})$, a value that is still observed after 10 min of motionless stance (D). These observations, combined with the low amplitudes that are observed, demonstrate that the stretchability of the applied long stretch bandage absorbs a certain amount of the gravitational venous filling forces that are related to the position change and allows volume changes of the included leg. However, these measurements also reveal that the applied force is high enough to counteract the forces responsible for the fluid shift into the interstitial tissue. This means that also extensible materials can play a role in the prevention of edema. ${ }^{15}$

\section{Conclusions}

It can be concluded that the SSI gives us a rough estimate of the effectiveness of an applied system but interpretation is influenced by the muscle forces of the person being bandaged as well as the measured effects of venous filling and, because of that, the timing of the measurements. However, the well-established SSI in general is able to differentiate between elastic and inelastic materials ${ }^{16}$ and the suggested cut-off point of 10 by the ICC, ${ }^{17}$ represents a very simple quotient that may be taken as a rule of thumb and is measurable in patients without major disfigurations of the legs due to severe obesity or lymphoedema. Future guidelines on measuring the SSI should include that the final standing pressure value should be taken when a stable recording over a certain period is observed.

\section{References}

1. Partsch H, Clark M, Bassez S, et al. Measurement of lower leg compression in vivo: recommendations for the performance of measurements of interface pressure and stiffness: a consensus statement. Dermatol Surg 2006;32:229-38.

2. European Committee for Standardization (CEN). Non-active medical devices. Working group 2 ENV 12718: European pre-standard medical compression hosie- ry. CEN TC 205. Brussels: CEN 2001.

3. Stolk R, Wegen van der-Franken CPM, Neumann HAM. A method for measuring the dynamic behavior of medical compression hosiery during walking. Dermatol Surg 2004;30:729-36.

4. Schuren J. Compression unravelled. Essen (Germany): Margreff Druck GmbH: 2011. p 134.

5. Partsch H. The static stiffness index: a simple method to assess the elastic property of compression material in vivo. Dermatol Surg 2005;31:625-30.

6. Veraart JCJM, Neumann HAM. Interface pressure measurements underneath elastic and non-elastic bandages. Phlebology 1996;1:S2-5.

7. Mosti G, Mattaliano V. Simultaneous changes of leg circumference and interface pressure under different compression bandages. Eur J Vasc Endovasc Surg 2007; 33:476-82.

8. Mosti G, Mattaliano V, Partsch H. Influence of different materials in multi-component bandages on pressure and stiffness of the final bandage. Dermatol Surg 2008;34:6319.

9. Partsch H, Clark M, Mosti G, et al. Classification of compression bandages: practical aspects. Dermatol Surg 2008;34: 600-9.

10. Nicolaides AN, Zukowski AJ. The value of dynamic venous pressure measurements. World J Surg 1986;10:919-24.

11. Eberhardt RT, Raffetto JD. Chronic venous insufficiency. Circulation 2005;111;2398409.

12. Pannier F, Rabe E. Optoelectric volume measurements to demonstrate volume changes in the lower extremities during orthostasis. Int Angiol 2010;29:395-400.

13. Stick C, Hiedl U, Witzleb E. Volume changes in the lower leg during quite standing and cycling exercise at different ambient temperatures. Eur J Appl Physiol 1993;66:427-33.

14. Mosti G, Mattaliano V, Partsch H. Inelastic compression increases venous ejection fraction more than elastic bandages in patients with superficial venous reflux. Phlebology 2008;23:287-94.

15. Stranden E. Edema in venous insufficiency. Phlebolymphol 2011;18:3-14.

16. Veraart JCJM, Daamen E, Neumann HAM. Short stretch versus elastic bandages: effect of time and walking. Phlebologie 1997;26:19-24.

17. Partsch H. The use of pressure change on standing as a surrogate measure of the stiffness of a compression bandage. Eur J Vasc Endovasc Surg 2005;30:415-21. 